\title{
Masas ováricas: Valoración con Doppler color transvaginal
}

\author{
Guido Parra A.; Eduardo Denubilla L.; Ricardo Torrents G.; Erika Hoyos C. *
}

\section{RESUMEN}

OBJETIVO: Adicionar un parámetro más en el diagnóstico de las masas ováricas que ayuden a su diferenciación (benignidad o malignidad) y por consiguiente a su manejo.

MATERIAL Y METODOS: Se incluyen 87 casos de masas ováricas diagnosticadas desde enero de 1993 hasta octubre de 1993 , mediante ecografía transvaginal Doppler-Color, usando un equipo ATL-Ultramak 9 DP y MDI-ESP provisto de traductores de 5, 7.5 y 9 mhz. La edad de las pacientes estaban comprendidas entre los 20-65 años.

RESULTADOS: 46 pacientes tuvieron resultados de anatomopatología (por video-Laparoscopia o Laparotomía); 3 casos fueron reportados como malignos (IR menor 0.45): 1 tumor de células de la granulosa (IR 0.43); 1 tumor seroso (Carcinoma Bordelline) (IR: 0.41$0.43)$; 1 cistadenocarcinoma (IR: 0.33 ).

Los restantes (43) presentaban índices de resistencia mayor de 0.45 y los reportes de anatomía patológica no mostraron malignidad. De los 41 pacientes sin estudios anatomapatológicos, 20 mostraron una desaparición de la masa en controles ecográficos posteriores concluyéndose que lo anterior se debe probablemente a que fueron quistes funcionales o cuerpos lúteos hemorrágicos.

CONCLUSIONES: De acuerdo con nuestro estudio consideramos que la valoración fluxométrica (índice Resistencia) es una prueba de mucha utilidad para la clasificación preoperatoria de malignidad o benignidad de las masas ováricas.

PALABRAS CLAVES: Masas ováricas, neoplasias malignas, neoplasias benignas, diagnóstico, doppler color.

SUMMARY

OBJECTIVE: To add another parameter for the diagnosis of the ovarian tumors in order to differentiate benign from malignant lesions and in consequence their management.

MATERIAL AND METHODS: 87 cases of ovarian tumors diagnosed by color doppler transvaginal ultrasonography, between january 1993 and october 1993 were included. We use an ATL-Ultramak 9 DP and MDI-ESP equipment with 5, 7.5 and 9 MHZ probes. The age range of the patients were between 20 and 65 years.

RESULTS: The histologic study was available in 46 patients (samples taken by video-laparoscopy or laparotomy); 3 cases were informed as malignant (RI < 0.45): One granulosa-cell tumor (RI: 0.43); one serous tumor (Borderline) (RI: 0.41-0.43) and one cystadenocarcinoma (RI: 0.33).

The other 43 showed resistance indexes $>0.45$ and the histologic studies didn't show malignancy. Of the 41 patients without pathologic study, the tumor disappear in 20 cases, These cases probably correspond to functional cysts or hemorrhagic luteal cysts.

CONCLUSIONS: Our results show that the fluxometric study (Resistance index) is a very useful test for the preoperatory classification of malignant an benign ovarian tumors.

KEY WORDS: Ovarian tumors, malignant, neoplasias, benign neoplasias, diagnosis, color doppler.

Dentro de las neoplasias malignas ginecológicas, el cáncer de ovario se ha constituido en los últimos años en el causante de la mayor mortalidad, especialmente en los países desarrollados (1).

Si bien su incidencia no es alta, comparativamente con otras neoplasias ginecológicas ( $\mathrm{Ca}$. de cuello, de endometrio, etc.), su alta mortalidad hace que su diagnóstico precoz cobre cada día mayor importancia y el

* Centro de Diagnóstico Ultrasonográfico, Clínica la Asunción, Barranquilla. hecho de no conocer con exactitud la etiología del mismo hace que no haya medidas preventivas contra él, como por ejemplo: Ca. pulmón - no fumar, Ca. mama autoexamen, etc.

Según algunas publicaciones entre el $20-30 \%$ de todos los $\mathrm{T}$. ováricos son malignos y el hecho que la esperanza de vida para las mujeres haya aumentado en los últimos años, hace que su incidencia teóricamente vaya a ser mayor.

Uno de los factores pronósticos más importantes, es el Estadio Tumoral cuando se realiza el diagnóstico, ya que más del $80 \%$ de ellos se diagnostican en estadio III- 
IV (2), según la clasificación FIGO (3). Si el aspecto teórico publicado, que el Doppler-Color agregado al US/ TV permite detectar flujos de bajas velocidades y al mismo tiempo puede valorar la presencia o ausencia de Angiogénesis, es cierto, su importancia estribaría en que dicha detección podría reconocer T. Malignos ya que este proceso de Angiogénesis sucede básicamente en ellos (4-5).

$\mathrm{Si}$ bien existen varias consideraciones generales sobre si el flujo debe medirse peritumoral o intratumoral, la idea es que la mayoría de los T. Malignos presentan flujo intratumoral, a diferencia de las masas benignas o quistes ováricos (6).

Además, es importante tener en cuenta la época del ciclo en que se realiza la medición: fase proliferativa o luteal, si la edad de la paciente examinada es prepuberal, fértil o post-menopáusica; sin embargo, el hecho de explorar la eficacia de un método no invasor como marcador tumoral es atractiva, especialmente en $\mathrm{T}$. de Ovario en donde los otros marcadores tumorales (Ca. 125, Alfafetoproteína, etc.), no han mostrado una mayor eficacia; especialmente en pacientes en edad reproductiva (7).

\section{Materiales y métodos}

En el período comprendido entre 1-02-93 al 31-09-93 se practicaron ecografías transvaginales en pacientes remitidas al centro, a las cuales se les diagnosticó la presencia de una masa anexial, practicándose además del análisis ya descrito para Eco-transvaginal (tiempo real, modo bidimensional) como criterios para establecer malignidad y benignidad (presencia de septum, grosor de la cápsula, ecogenicidad, presencia de ascitis, etc.), también se practicó una medición del Indice de Resistencia (I/R: S-D/S) de las arterias que estuvieran tanto en la zona peritumoral como en la intratumoral, escogiéndose el de menor valor como el índice a anotar.

Las pacientes en edad fértil, se les realizó la Ecografía Transvaginal en la fase folicular y en aquellas que estaban tomando anticonceptivos o se encontraban en la postmenopausia, se les realizó el día en que fueron remitidas.

El valor del índice no fue reportado ni tomado como referencia por la decisión clínica y/o terapéutica.

Las ecografías transvaginales y las mediciones de los índices se realizaron con equipos ATL-ultramar 9DP y 9HDI, con transductores transvaginales con frecuencia de 5 a 9 MHZ.

\section{Resultados}

Las 87 pacientes tuvieron edades comprendidas entre los 20 y los 65 años, para un promedio de 34.6 (tabla 1) y 46 de ellas fueron llevadas a cirugía por su médico tratante obteniéndose resultado de anatomía patológica en todas; de las 41 restantes, 28 fueron remitidas posteriormente a control ecográfico en los 4 meses que siguieron de la ecografía inicial encontrándose en 20 de ellas desaparición de la masa inicial y en las otras 8 hubo persistencia de la misma (ver tabla 2); y los índices de resistencia variaron entre lapso de 0.33 hasta 0.93 (ver tabla 3) y los tumores malignos estuvieron por debajo del índice de 0.45 sólo en el caso No. 25 que presentó un Indice de Resistencia igual a 0.41 y no se obtuvo información del acto quirúrgico ni de su anatomía patológica.

El tamaño de las masas no se relacionó con el resultado de malignidad ya que ellos se distribuyeron arbitrariamente en los grupos (ver tabla 4) al igual que tampoco fue relevante su asociacíón con la edad (ver tabla 2) de los pacientes. Hubo indistintamente en las masas malignas características de cápsula gruesa, delgada o irregular, al igual sus otros signos no demostraron asociación.

Entre los benignos, la distribución fue de 19 endometriomas, 5 quistes hemorrágicos, 10 dermoides, 2 cistoadenomas mucinoso, 1 torsión ovárica, 1 cistoadenoma seroso, 1 hidrosalpinx, 4 abscesos tubováricos, los cuales todos presentaron índices mayores de 0.45 .

Aplicando análisis de valores pronósticos encontramos que con índices de resistencias < de 0.45 los resultados con nuestros casos se van a $100 \%$, lo que indicaría este índice como el ideal; pero si usamos 0.40 como nivel de corte la sensibilidad baja de 33,3 y valor predictivo negativo al $97.4 \%$ (ver tabla 5).

\section{Discusión}

El análisis de los datos nos hace pensar que existe una clara diferencia entre la tendencia a índices de resistencia altos en los procesos benignos y una tendencia a índices bajos en los procesos malignos, o en los casos de procesos inflamatorios lo cual señalaría la baja resistencia al flujo de los vasos de ambas entidades; la discusión central estaría dada en cuál índice aceptar como punto de corte entre malignidad y benignidad (8), con buenos resultados en términos de sensibilidad y especificidad. $\mathrm{Si}$ lo miramos a la luz de nuestros resultados indudablemente 0,45 sería el ideal, pero series mayores como la de Kurjak señalan 0,40 (9) como el mejor; teóricamente pensamos que otros valores derivados de la onda de flujo tales como índice de pulsatilidad y/o S/D podrían conformar una sumatoria de puntajes con un valor específico que sería el que inclinara la balanza para pensar en criterios de malignidad. El otro punto a discutir es el tamaño de las masas malignas detectadas, la menor fue de 2-3 cms. y el estadio menor de clasificación tumoral al momento del acto quirúrgico (de acuerdo con la clasificación internacional de la FIGO) fue de IA (caso No. 29) tumor seroso "Border Line" y los demás estadios IB e IC, es conocido que la neovascularización es determinante en el crecimiento tumoral después de 3-4 mm., esto sería una base para el rastreo sistemático de toda masa detectada por ecografía transvaginal y nos haría pensar en la posibilidad que este método se emplee como método de "screening", cuestión que estaría por demostrar en estudios de mayor envergadura (10). Ante la imposibilidad de obtener los resultados del cuarto caso como índice por debajo de 0.45 (caso No. 25) podríamos asumir que aún siendo benigno no cambiaría significativamente los resultados en términos de valor pronóstico o sensibilidad-especificidad. 
Tabla 1

DISTRIBUCION GENERAL MASAS OVARICAS

\begin{tabular}{|c|c|c|c|c|c|c|c|c|c|c|}
\hline Caso & Edad & $\mathbf{T A}$ & $\mathbf{S}$ & $\mathbf{C}$ & $\mathbf{E}$ & $\mathbf{A}$ & $\mathbf{I} / \mathbf{R}$ & Patología & CTR. & RE. \\
\hline 1 & 22 & 2.5 & 0 & G & $\mathrm{H}$ & 0 & 0.62 & Endometrioma & & \\
\hline 2 & 20 & 4 & 1 & $\mathrm{G} / \mathrm{I}$ & M & 0 & 0.69 & Dermoide & & \\
\hline 3 & 31 & 3 & 0 & G/D & $\mathrm{H}$ & 0 & 0.72 & Endometrioma & & \\
\hline 4 & 27 & 3 & 0 & D & $\mathrm{H}$ & 1 & 0.8 & Q. Hemorrágico & & \\
\hline$* 5$ & 33 & 12 & 1 & $\mathrm{D} / 1$ & A & 1 & 0.43 & T.C. Granulosa & & \\
\hline 6 & 29 & 3.5 & 0 & $\mathrm{G} / 1$ & $\mathrm{H}$ & 0 & 0.68 & Dermoide & & \\
\hline 7 & 40 & 10 & 0 & $\mathrm{D}$ & A & 0 & 0.72 & & $S$ & 0 \\
\hline 8 & 52 & 6 & 0 & $\mathrm{D}$ & A & 0 & 0.56 & & S & 0 \\
\hline 9 & 22 & 2.5 & 0 & G & $\mathrm{H}$ & 1 & 0.49 & & $S$ & 1 \\
\hline 10 & 39 & 4 & 0 & G & M & 0 & 0.77 & Endometrioma & & \\
\hline 11 & 31 & 3.5 & 0 & $\mathrm{D}$ & $\mathrm{H}$ & 0 & 0.63 & & $S$ & 0 \\
\hline 12 & 30 & 2.5 & 0 & G & M & 0 & 0.66 & Dermoide & & \\
\hline 13 & 27 & 7 & 0 & G & M & 1 & 0.52 & Abs. tuboovárico & & \\
\hline 14 & 42 & 5 & 0 & $\mathrm{D}$ & A & 0 & 0.79 & & $\mathrm{~S}$ & 0 \\
\hline 15 & 20 & 5 & 0 & $\mathrm{D}$ & $\mathrm{H}$ & 1 & 0.61 & & $S$ & 0 \\
\hline 16 & 40 & 6 & 1 & $\mathrm{D}$ & $\mathrm{H}$ & 0 & 0.7 & Cis. Mucinoso & & \\
\hline 17 & 42 & 4 & 0 & G & $\mathrm{H}$ & 0 & 0.68 & & $S$ & 1 \\
\hline 18 & 30 & 4.5 & 0 & $\mathrm{D}$ & $\mathrm{H}$ & 0 & 0.69 & Q. Hemorrágico & & \\
\hline 19 & 31 & 2.5 & 0 & $\mathrm{G}$ & $\mathrm{H}$ & 0 & 0.71 & & $\mathrm{~N}$ & \\
\hline 20 & 39 & 3 & 0 & G & $\mathrm{H}$ & 0 & 0.73 & Endometrioma & & \\
\hline 21 & 32 & 7 & 0 & $\mathrm{D}$ & A & 0 & 0.73 & & $\mathrm{~N}$ & \\
\hline 22 & 41 & 6.6 & 1 & G & M & 0 & 0.49 & Abs. tuboovárico & & \\
\hline 23 & 61 & 3.5 & 0 & $\mathrm{D}$ & A & 0 & 0.6 & & $\mathrm{~S}$ & 1 \\
\hline 24 & 29 & 5.5 & 0 & G & M & 0 & 0.75 & Dermoide & & \\
\hline 25 & 50 & 10.5 & 1 & $\mathrm{G} / \mathrm{l}$ & M & 1 & 0.41 & & $\mathrm{~N}$ & \\
\hline 26 & 65 & 6 & 0 & $\mathrm{D}$ & A & 0 & 0.63 & & $S$ & 0 \\
\hline 27 & 30 & 4 & 0 & $\mathrm{G}$ & M & 0 & 0.73 & Dermoide & & \\
\hline 28 & 29 & 2.5 & 0 & G & $\mathrm{H}$ & 0 & 0.75 & & $S$ & 0 \\
\hline$* 29$ & 41 & 2.5 & 0 & G & $\mathrm{S}$ & 0 & 0.42 & T. Seroso B.L. & & \\
\hline 30 & 22 & 2.5 & 0 & G & $\mathrm{H}$ & 0 & 0.79 & Endometrioma & & \\
\hline 31 & 24 & 4 & 0 & $\mathrm{D}$ & A & 0 & 0.71 & $\mathrm{~S}$ & 0 & \\
\hline 32 & 26 & 4.5 & 0 & G & $\mathrm{H}$ & 0 & 0.63 & & $\mathrm{~S}$ & 0 \\
\hline 33 & 28 & 6 & 0 & $\mathrm{D}$ & A & 0 & 0.8 & Hidrosalpin $x$ & & \\
\hline 34 & 33 & 3.5 & 0 & $\mathrm{D} / \mathrm{I}$ & $\mathrm{H}$ & 0 & 0.53 & & $\mathrm{~S}$ & 1 \\
\hline 35 & 43 & 5 & 0 & $\mathrm{D}$ & A & 0 & 0.69 & & $\mathrm{~S}$ & 0 \\
\hline 36 & 41 & 2.5 & 0 & G & $\mathrm{H}$ & 0 & 0.63 & & $\mathrm{~N}$ & \\
\hline 37 & 28 & 3 & 1 & G & $\mathrm{H}$ & 1 & 0.79 & Endometrioma & & \\
\hline 38 & 20 & 3.5 & 0 & G & $\mathrm{H}$ & 0 & 0.66 & Endometrioma & & \\
\hline 39 & 25 & 8.5 & 1 & G & $\mathrm{H}$ & 1 & 0.51 & Abs. tuboovárico & & \\
\hline 40 & 38 & 7 & 1 & G & $\mathrm{H}$ & 1 & 0.48 & & $\mathrm{~N}$ & \\
\hline 41 & 39 & 4 & 0 & $\mathrm{D}$ & A & 0 & 0.57 & & $\mathrm{~S}$ & 0 \\
\hline 42 & 26 & 2 & 0 & $\mathrm{G}$ & M & 0 & 0.75 & Endometrioma & & \\
\hline 43 & 21 & 3.5 & 1 & $\mathrm{G}$ & $\mathrm{H}$ & 1 & 0.82 & Q. Hemorrágico & & \\
\hline 44 & 48 & 3.5 & 0 & G & $\mathrm{H}$ & 0 & 0.69 & Endometrioma & & \\
\hline 45 & 29 & 2.8 & 0 & $\mathrm{G} / \mathrm{l}$ & $\mathrm{H}$ & 0 & 0.67 & Endometrioma & & \\
\hline 46 & 43 & 3 & 0 & G & $\mathrm{H}$ & 0 & 0.56 & & $\mathrm{~S}$ & 0 \\
\hline 47 & 31 & 4.5 & 0 & $\mathrm{D}$ & A & 0 & 0.63 & & $\mathrm{~N}$ & \\
\hline 48 & 32 & 5.5 & 0 & $\mathrm{D}$ & A & 0 & 0.65 & & $\mathrm{~N}$ & \\
\hline
\end{tabular}


Continúa Tabla 1

\begin{tabular}{|c|c|c|c|c|c|c|c|c|c|c|}
\hline Caso & Edad & TA & $\mathbf{S}$ & C & $\mathbf{E}$ & $\mathbf{A}$ & $\mathbf{I} / \mathbf{R}$ & Patología & CTR. & RE. \\
\hline 49 & 43 & 7 & 1 & D & A & 1 & 0.59 & Cis. Seroso & & \\
\hline 50 & 33 & 3.5 & 0 & $\mathrm{G} / \mathrm{I}$ & $\mathrm{H}$ & 0 & 0.72 & Endometrioma & & \\
\hline 51 & 37 & 2 & 0 & G & M & 0 & 0.68 & Dermoide & & \\
\hline 52 & 26 & 4.5 & 0 & $\mathrm{G} / \mathrm{I}$ & A & 1 & 0.93 & Torsión ovario & & \\
\hline 53 & 47 & 4 & 0 & $\mathrm{D}$ & M & 0 & 0.83 & & $S$ & 0 \\
\hline 54 & 31 & 4.5 & 0 & G & M & 0 & 0.71 & Endometrioma & & \\
\hline 55 & 29 & 4 & 0 & $\mathrm{G}$ & $\mathrm{M}$ & 0 & 0.74 & Dermoide & & \\
\hline 56 & 25 & 6 & 0 & D & A & 0 & 0.65 & & $\mathrm{~S}$ & 0 \\
\hline 57 & 20 & 2 & 0 & D & $\mathrm{H}$ & 0 & 0.53 & & $\mathrm{~N}$ & \\
\hline 58 & 28 & 5 & 1 & G & M & 0 & 0.63 & Endometrioma & & \\
\hline 59 & 36 & 2 & 0 & $\mathrm{G}$ & $\mathrm{H}$ & 0 & 0.74 & & $\mathrm{~N}$ & \\
\hline 60 & 40 & 8 & 1 & G & $\mathrm{H}$ & 1 & 0.61 & Cis. Mucinoso & & \\
\hline 61 & 36 & 3.5 & 0 & G & $\mathrm{H}$ & 1 & 0.79 & Q. Hemorrágico & & \\
\hline 62 & 52 & 2 & 0 & $\mathrm{D}$ & A & 0 & 0.77 & & $\mathrm{~S}$ & 0 \\
\hline 63 & 37 & 4 & 0 & G & $\mathrm{H}$ & 0 & 0.79 & Endometrioma & & \\
\hline 64 & 21 & 3 & 0 & $\mathrm{G} / \mathrm{I}$ & M & 0 & 0.62 & Dermoide & & \\
\hline 65 & 36 & 3.8 & 0 & G & M & 0 & 0.68 & Endometrioma & & \\
\hline 66 & 33 & 4.6 & 1 & G & $\mathrm{H}$ & 0 & 0.72 & Endometrioma & & \\
\hline 67 & 60 & 4 & 0 & $\mathrm{D}$ & A & 0 & 0.79 & & $S$ & 1 \\
\hline 68 & 28 & 13.5 & 0 & G & $S$ & 1 & 0.51 & & $\mathrm{~N}$ & \\
\hline 69 & 41 & 80 & 0 & $\mathrm{D}$ & A & 0 & 0.73 & & $S$ & 0 \\
\hline 70 & 27 & 11 & 1 & $\mathrm{G} / \mathrm{I}$ & M & 0 & 0.55 & Abs. Tuboovárico & & \\
\hline 71 & 31 & 4.5 & 0 & G & $\mathrm{H}$ & 0 & 0.49 & & $S$ & 0 \\
\hline 72 & 35 & 3.5 & 0 & $\mathrm{G}$ & M & 0 & 0.65 & Dermoide & & \\
\hline 73 & 34 & 13 & 1 & G & A & 0 & 0.47 & & $\mathrm{~N}$ & \\
\hline 74 & 25 & 3.5 & 1 & $\mathrm{G} / \mathrm{I}$ & $\mathrm{H}$ & 0 & 0.81 & Q. Hemorrágico & & \\
\hline 75 & 50 & 2.5 & 0 & $\mathrm{D}$ & A & 1 & 0.57 & & $S$ & 1 \\
\hline 76 & 32 & 5.5 & 0 & G & $\mathrm{H}$ & 0 & 0.7 & Endometrioma & & \\
\hline 77 & 42 & 14 & 0 & G & $\mathrm{H}$ & 1 & 0.63 & & $S$ & 1 \\
\hline 78 & 61 & 6.5 & 0 & $\mathrm{D}$ & A & 1 & 0.73 & & $S$ & 1 \\
\hline 79 & 57 & 4 & 0 & $\mathrm{D}$ & A & 0 & 0.68 & & $S$ & 0 \\
\hline$* 80$ & 59 & 5 & 1 & $\mathrm{D} / \mathrm{I}$ & $\mathrm{M}$ & 0 & 0.33 & Cistoadenoca. & & \\
\hline 81 & 24 & 3 & 0 & G & $\mathrm{H}$ & 0 & 0.77 & Endometrioma & & \\
\hline 82 & 35 & 3.5 & 0 & G & M & 0 & 0.53 & & $\mathrm{~N}$ & \\
\hline 83 & 28 & 4 & 0 & $\mathrm{G} / \mathrm{I}$ & M & 1 & 0.64 & Dermoide & & \\
\hline 84 & 25 & 6 & 0 & D & A & 0 & 0.71 & & $\mathrm{~S}$ & 0 \\
\hline 85 & 22 & 3.5 & 0 & $\mathrm{D}$ & A & 0 & 0.77 & & $\mathrm{~N}$ & \\
\hline 86 & 42 & 3.5 & 1 & $\mathrm{D}$ & $\mathrm{H}$ & 0 & 0.74 & Endometrioma & & \\
\hline 87 & 20 & 4 & 0 & $\mathrm{D}$ & A & 0 & 0.81 & & $\mathrm{~S}$ & 0 \\
\hline Suma & 3010 & 419.8 & 18 & & & 19 & 57.61 & & & \\
\hline $\mathrm{X}$ ? 3 & 34.5977011 & 4.825287 & & & & 839 & 04598 & & & \\
\hline MAX & 65 & 14 & & & & & 0.93 & & & \\
\hline MIN & & 20 & 2 & & & & & 0.33 & & \\
\hline
\end{tabular}

$\mathrm{TA}=$ Tamaño masa $\mathrm{S}=$ Septum

$\mathrm{C}=$ Cápsula $\quad(\mathrm{G}=$ Gruesa $\mathrm{D}=$ Delgada $\mathrm{I}=$ Irregular $)$

$\mathrm{E}=$ Ecogenicidad $(\mathrm{H}=$ Hipoecogénica $\mathrm{A}=$ Aneroica $\mathrm{M}=$ Mixta $\mathrm{S}=$ Sólida $) \quad \mathrm{RE}=$ Resultado del control

$\mathrm{A}=$ Ascitis $\quad *=\mathrm{T}$. Malignos CTR.= Control. 


\section{Conclusión}

Consideramos que el uso del índice fluxométrico de resistencia $(S-D) / S)$, es consistente en señalar estadios de malignidad con un índice tope de 0,45 para tumores ováricos y que podría constituirse en pilar importante en la detección precoz del cáncer ovárico.

Posteriores refinamientos de esta técnica Doppler Color, como el uso de agentes contrastantes para ultrasonido, permiten todavía detectar aún más precozmente vascularización con características de angiogénesis.

Tabla 2

\section{DISTRIBUCION SEGUN EDAD}

\begin{tabular}{|ccc|}
\hline Edad & F & T. Malignos \\
\hline $15-25$ & 5 & \\
$26-30$ & 12 & \\
$31-35$ & 19 & 1 \\
$36-40$ & 16 & \\
$41-45$ & 12 & 1 \\
$46-50$ & 11 & \\
$51-55$ & 4 & 1 \\
$56-60$ & 2 & \\
$61-65$ & 3 & \\
$66-70$ & 3 & \\
Total & 87 & \\
\hline
\end{tabular}

Tabla 3

DISTRIBUCION SEGUN I/R

\begin{tabular}{|lrc|}
\hline \multicolumn{1}{|c}{ I/R } & F & T. Malignos \\
\hline $0.23-0.33$ & 1 & 1 \\
$0.34-0.43$ & 3 & 2 \\
$0.44-0.53$ & 11 & \\
$0.54-0.63$ & 18 & \\
$0.64-0.73$ & 31 & \\
$0.74-0.83$ & 22 & \\
$0.84-0.93$ & 1 & \\
Total & 87 & \\
\hline
\end{tabular}

Tabla 4

DISTRIBUCION SEGUN TAMAÑO

\begin{tabular}{|lrc|}
\hline Tamaño & F & T. Malignos \\
\hline $1-2$ & 5 & 1 \\
$3-4$ & 45 & \\
$5-6$ & 21 & 1 \\
$7-8$ & 8 & \\
$9-10$ & 2 & 1 \\
$11-12$ & 2 & \\
$13-14$ & 3 & \\
Total & 87 & \\
\hline
\end{tabular}

Tabla 5

COMPARACION INDICES DE RESISTENCIA

\begin{tabular}{|c|c|c|c|c|c|c|}
\hline \multicolumn{4}{|c|}{$<0.45$} & \multicolumn{3}{|c|}{$<0.4$} \\
\hline & Malig. & Ben & Total & Malig. & Ben & Total \\
\hline $\mathrm{Si}$ & 3 & 0 & 3 & 1 & 0 & 1 \\
\hline \multirow[t]{2}{*}{ No } & 0 & 83 & 83 & 2 & 83 & 85 \\
\hline & 3 & 83 & 86 & 3 & 83 & 86 \\
\hline V.P.+ & & & 100 & & & 100 \\
\hline V.P.- & & & 100 & & & 97.4 \\
\hline SEN & & & 100 & & & 33.3 \\
\hline ESPE & & & 100 & & & 100 \\
\hline PRES & & & 100 & & & 97.6 \\
\hline
\end{tabular}

\section{BIBLIOGRAFIA}

1. Silberg E., Bonng C., Squires T. Cáncer Stadistics. Ca 40; 90, 1990.

2. Bourne TH., Whitehead MI., Campbell S. et.d. Ultra Sound Screening for Faminal Ovarian Cancer. Ginecol. Oncol. 1991; 43: 89-91.

3. FIGO. Cancer Committe: Staging anouncement, Ginecol. Oncol. 1986; $25: 383$.

4. Bourne T., Campbell S., Steer C. Whitehead MI and Collins WP. Transvaginal colour flow imaging: a posible new screening technique for ovarian cancer. Br. Med. J. 1989; 299: 1367.5.

5. Folkman J. Tumor Angiogénesis. Adv. Cancer Res. 1985; 43: 175.

6. Van Nagell Jr., Higgins RV., Donaldoon ES et al. Transvaginal Sonographic as a screening test for ovarian cancer. A report of the fist 1000 cases screened. Cancer 1990; 65: 93-577.
7. Finkler NJ., Benocerrag B., Javin P et al. Comparison of serum CA 125 Clinical impression, and ultrasound in the properative evaluation of ovarian masses. Obstet. Gynecol. 1989; 96: 1021-1023.

8. Kawai M., Kam T., Kikkawa F et al. Transvaginal Doppler Ultrasound with Color flow imaging in the diagnosis of ovarian cancer. Obstet. Gynecol. 1992; 79: 163-167.

9. Kurjak A. Transvaginal color Doppler. Carnforth, New Gessey Parthenon Publishing 1990.

10. Fleischer A., Jones H. Early Detection of ovarian carcinoma with transvaginal Sonography Potential and Limitations, New York, Raven Press, 1993. 